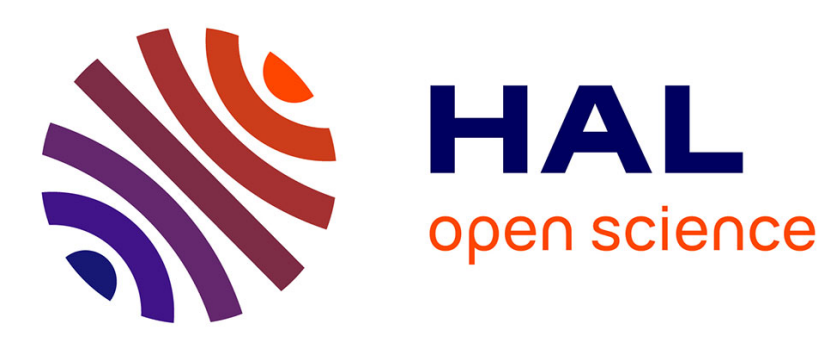

\title{
La culture en action des enseignants
}

Marc Durand, Luc Ria, Eric Flavier

\section{To cite this version:}

Marc Durand, Luc Ria, Eric Flavier. La culture en action des enseignants. Revue des sciences de l'education, 2002, 28 (1), pp.83-103. 10.7202/007150ar . hal-00803997

\section{HAL Id: hal-00803997 \\ https://hal.science/hal-00803997}

Submitted on 24 Mar 2013

HAL is a multi-disciplinary open access archive for the deposit and dissemination of scientific research documents, whether they are published or not. The documents may come from teaching and research institutions in France or abroad, or from public or private research centers.
L'archive ouverte pluridisciplinaire HAL, est destinée au dépôt et à la diffusion de documents scientifiques de niveau recherche, publiés ou non, émanant des établissements d'enseignement et de recherche français ou étrangers, des laboratoires publics ou privés. 


\title{
LA CULTURE EN ACTION DES ENSEIGNANTS
}

\author{
\begin{tabular}{c} 
REVUE DES SCIENCES DE L'EDUCATION (2002) \\
Revue des Sciences de I'Éducation, VOL. XXVIII, 1, 83-103. \\
\hline
\end{tabular} \\ Marc Durand*, Luc Ria**, Eric Flavier* \\ * Equipe ESTEREL, Institut Universitaire de Formation des Maitres \\ Montpellier, France \\ ** Laboratoire d'Anthropologie des Pratiques Corporelles, UFR STAPS Clermont-Ferrand, France
}

\begin{abstract}
Résumé
Dans une perspective d'action située, cet article propose une analyse de la cognition et de la culture en action des enseignants. La première partie porte sur les processus d'actualisation et de construction des connaissances dans l'action ; la deuxième sur les relations en apparence contradictoires entre le caractère singulier des actions en classe et la possibilité d'une culture partagée ; la troisième sur les processus de transmission au sein de cette communauté des pratiques enseignantes.
\end{abstract}

Mots clés

Action située, Enseignant, Culture professionnelle, Cognitions

TEACHERS' ENACTED CULTURE

Summary

From a situated action perspective, this article aims to account for teachers' cognition and enacted culture. The first part deals with the process of enacted knowledge actualization and construction ; the second part with the relationships between the singularity of the classroom action and the possibility of a shared culture ; the third part with the transmission process within the teachers community of practice.

Key words

Situated Action, Teachers, Professional Culture, Cognition 
Dans son ouvrage de 1988, consacré à la cognition “ dans la vie de tous les jours ”, Jean Lave plaidait pour un programme de recherche en anthropologie cognitive, positionné entre deux naïvetés : celle des sociologues lorsqu'ils s'intéressent à la cognition, celle des psychologues lorsqu'ils s'intéressent à la culture. Cet article est une tentative de soutenir le défi de rendre compte simultanément de la cognition et de la culture des enseignants.

Cognition et culture sont ici envisagées en action et en situation, c'est à dire au cours de pratiques réelles. Cette perspective pragmatique est ancrée sur deux présupposés principaux. Le premier est que les notions de culture et de cognition sont assimilables à des processus de construction ou d'exploitation de significations dans l'accomplissement de l'action quotidienne (Theureau, 1992 ; Whitson, 1997). Rendre compte de la culture ou des cognitions d'un acteur implique de reconstituer ces processus sémiotiques. Le deuxième est que la cognition est essentiellement située, c'est à dire nichée dans un contexte social, spatio-temporel, corporel dont elle est partiellement l'expression et qu'elle contribue à définir et façonner (Brown, Collins et Duguid, 1998 ; Greeno, 1998 ; Hutchins, 1995 ; Kirshner et Whitson, 1997 ; Lave, 1988 ; Suchman, 1987). Toute communauté professionnelle est porteuse d'une culture, ensemble de savoirs, de valeurs, de normes qui formatent les actions professionnelles et qu'en retour ces dernières contribuent à faire vivre et évoluer (Becker et Carper, 1956 ; Becker, Geer, Riesman et Weiss, 1968 ; Dubar et Tripier, 1998 ; Hughes, 1958). Les éléments constitutifs de la culture des enseignants sont par conséquent repérables au cœur même de l'action individuelle et l'ambition de cet article est d'illustrer quelques traits saillants de cette culture en action. Il s'appuie sur des données obtenues auprès d'enseignants à différents niveaux d'acculturation professionnelle ${ }^{1}$. Certains sont expérimentés, d'autres novices, d'autres encore maîtres de stages (ou conseillers pédagogiques), c'est à dire des enseignants expérimentés ayant notamment pour mission de transmettre cette culture aux novices.

Trois axes sont développés. Le premier, à partir de l'analyse de la prise en main d'une classe par un enseignant expérimenté, développe la notion même de culture en action et insiste sur les processus d'actualisation et de construction située de la culture individuelle. Le deuxième concerne la singularité de l'action des enseignants et discute de la possibilité de développer une communauté de pratiques ; quelques extraits d'interactions entre maîtres de stage en formation initiale des enseignants illustrent l'idée d'une structuration de cette communauté de pratiques basée sur la distinction entre genres collectifs et styles personnels. Le troisième concerne la dynamique de cette culture conçue comme vivante et évolutive ; des stratégies et dispositifs de formation initiale sont envisagés comme des situations privilégiées permettant d'illustrer certains processus collectifs par lesquels elle se développe, se transmet, se propage.

\footnotetext{
1 Ces données sont extraites de compte rendus de recherches, notamment :

Durand, M. (2000). Développement personnel et accès à une culture professionnelle en formation initiale des professeurs. In C. Gohier et C. Alin, (Eds), Enseignant-Formateur. La construction de l'identité professionnelle (pp. 67-83). Paris : L'Harmattan.

Durand, M. (2001). Chronomètre et survêtement : Reflets de l'expérience quotidienne d'enseignants d'Education Physique. Paris : Editions Revue EPS.

Ria, L. (2001). Les préoccupations des enseignants débutants en Education Physique et Sportive. Etude de l'expérience professionnelle et conception d'aides à la formation. Thèse de doctorat STAPS non publiée. Université de Montpellier 1.

Ria, L., Saury, J., Sève, C., Durand, M. (2001). Les dilemmes des enseignants débutants : Etudes lors des premières expériences de classe en Education Physique. Science et Motricité, 42, 47-58.
} 
La culture en action des enseignants

Cognition culturelle et culture en action

De la cognition culturellement située à la culture en action

Les présupposés classiques au sein du programme naturaliste en sciences de la cognition sont désormais sérieusement discutés (Dreyfus, 1986 ; Greeno, 1998 ; Still et Costall, 1991 ; Varela, 1989 ; Winograd et Florès, 1989). Les arguments pour une approche culturaliste de la cognition sont nombreux et convaincants. Quelques-uns sont envisagés ci-dessous.

A l'encontre de l'idée selon laquelle les modes de pensée humains auraient un caractère d'universalité ou d'invariance, de nombreuses observations ont mis en évidence le fait que les raisonnements sont étroitement liés aux contextes de leur déploiement et ne peuvent être restitués sans une formalisation rigoureuse de ces contextes (Lave, 1988 ; Lave et Wenger, 1991 ; Suchman, 1987). Cette nécessité de prendre en compte de façon centrale le contexte de l'action concerne les secteurs de l'industrie (Engeström et Middelton, 1996 ; Leplat, 2000 ; Theureau, 1992) ou de l'école et de la formation (Bredo, 1994 ; Brown, Collins et Duguid, 1989 ; Greeno, 1998 ; Gal-Petitfaux et Durand, 2001 ; Lave, 1988 ; Leblanc, Saury, Sève, Durand et Theureau, 2001).

Les contextes de l'action sont envisagés à différentes échelles, de la plus large à la plus restreinte. A un plan large, chaque culture constitue un creuset au sein duquel s'élaborent et se transmettent des procédures, normes, valeurs qui documentent ou formatent les modes de pensée et d'action : par exemple, des navigateurs trukese des îles polynésiennes ne procèdent pas de la même façon que des marins occidentaux pour se repérer en mer (Hutchins, 1995). Dans une acception plus réduite, le contexte désigne des conditions d'action spécifiques : par exemple un même acteur résout différemment des problèmes d'arithmétique isomorphes selon le contexte dans lequel ils sont posés (Carraher, Carraher et Schliemann, 1985 ; Lave, 1988 ; Schliemann et Carraher, 1992). Au plan professionnel, en un sens encore plus délimité, au sein même d'un atelier s'expriment des habitudes, des manières de faire, sentir, penser que le novice doit assimiler pour s'intégrer dans chaque communauté de pratiques et devenir professionnel (Chaiklin et Lave, 1991 ; Clot, 1999 ; Lave, 1988).

Le contexte de l'action ne reçoit pas dans le cadre de cette approche de la cognition située une définition naturaliste, positive. Il ne s'agit pas d'un monde objectif, mais de mondes personnels, construits ou re-construits à chaque instant par l'acteur selon les circonstances présentes de son engagement (Durand et Arzel, sous presse ; Quéré, 2000 ; Suchman, 1987 ; Theureau, 1992 ; Varela, 1989). Par conséquent, la cognition est étudiée comme un phénomène culturel, c'est à dire dont les contenus et les processus contribuent à constituer et à exprimer une culture. Notre analyse cherche à saisir ce qui dans chaque acte professionnel, au plus précis des dynamiques individuelles, est l'expression d'une culture.

La culture est donc appréhendée au niveau des acteurs, comme un ensemble d'unités cognitives utiles pour "l'action qui convient" au sens où elle satisfait des normes et valeurs d'un groupe social constitué, et des contraintes d'efficacité en vue de l'atteinte d'un but (Thévenot, 1990). Ces éléments constitutifs de la culture individuelle sont des composantes des expériences passées des acteurs, condensées et convoquées dans l'action ici et maintenant (Clot, 1999 ; Theureau, 2000). A chaque instant l'action récapitule, réactualise la culture de l'acteur qui n'est pas un système clos, stable et inerte, mais un ensemble vivant mobilisé et modifié potentiellement de façon plus ou moins durable et forte. La culture de chaque acteur est donc de nature mémorielle et expériencielle et revêt un caractère intime, singulier, en étroite connexion avec sa biographie, tout en étant aussi fondamentalement ce qu'il y a de partagé ou de partageable dans le répertoire de cet acteur. Ceci suppose que les expériences individuelles dépassent leurs propres occurrences et soient généralisées ou généralisables (Quéré, 2000). A titre d'hypothèse, nous considérons que cette généralisation repose sur des processus de typicalisation ou de typification des expériences individuelles (Rosch, 1973 ; Varela, Thompson et 
Rosch, 1993 ; Theureau, 1992) : l'action est l'expression et la mise en œuvre d'une typicalisation créatrice de la culture à laquelle l'acteur appartient.

La culture en action présente donc un double visage qui pourrait paraître paradoxal, en ce sens qu'elle revêt un caractère éminemment singulier, individuel à l'exacte échelle de l'idiosyncrasie des actions humaines, tout en étant l'expression de traits collectifs, sociaux et partagés (Quéré, 2000).

\section{$\underline{\text { Semiosis et culture en action des enseignants }}$}

Notre analyse s'inscrit dans une approche sémiologique de l'action humaine (Theureau 1992, 2000 ; Whitson, 1997) qui est exposée à grands traits dans les lignes qui suivent.

L'action est considérée comme sécable en unités élémentaires qui sont des unités significatives pour l'acteur. Chacune de ces unités est envisagée comme l'expression d'une activité sémiotique complexe, d'une semiosis appréhendée à partir d'une ré-interprétation des concepts développés par Peirce $(1978$; 1984). Ces unités participent plus globalement au flux continu de signification que l'acteur attribue à son engagement au monde.

L'action est indéterminée, non réductible à l'exécution d'un plan : c'est une improvisation permanente. Cette improvisation est cadrée par l'intention de l'acteur, sur un mode vague et ouvert. C'est dans l'occurrence même de cette action que les composantes signifiantes de l'expérience humaine se précisent et prennent forme. Il est théoriquement possible de décrire à chaque pas, pour chaque unité élémentaire les processus fondamentaux constitutifs de cette semiosis $^{2}$. Ces processus sont illustrés ici à partir d'un extrait du début d'une leçon de Français animée par un enseignant expérimenté (10 années d'ancienneté) dans la classe de première année d'un lycée de centre ville (élèves de 15 ans) au retour d'une période de vacances, au mois de février.

Professeur : “ Bonjour à tous. J'espère que vous avez profité de ces quelques jours de vacances pour vous reposer et reconstituer votre force de travail. Vous en aurez besoin parce que l'échéance de la fin du trimestre approche. Il ne reste qu'un petit mois d'ici au prochain conseil de classe qui, comme vous le savez, est très important pour votre orientation. Vous allez sans doute penser que j'insiste lourdement, mais je vous rappelle que pour vous, ce petit mois est très important. Il vous faut prendre en main votre destin si j'ose dire... Et soigner votre bulletin scolaire. Pour cela il n'y a pas d'autre solution qu'un travail assidu et régulier. Vous savez que les enseignants sont là pour vous aider... En tout cas n'hésitez pas à me solliciter... Je suis là pour vous aider à réaliser vos projets..."

Auto-confrontation : “ C'est le début du cours et la reprise après les vacances. C'est un moment particulier. Je sais qu'il y a une tendance au relâchement chez la plupart des élèves au deuxième trimestre et quand les beaux jours reviennent. Là je le vois tout de suite en entrant : ils ne sont pas dans le coup, certains d'entre eux... Bronzés et tout excités de se retrouver... Je me suis dit qu'il fallait... Gentiment bien sûr, mais qu'il fallait les remettre tout de suite dans la dure réalité. J'évite généralement d’être grandiloquent... On sait

\footnotetext{
${ }^{2}$ Pour décrire ce niveau de l'expérience qui est significatif pour l'acteur, c'est-à-dire montrable, racontable et commentable par lui à tout instant de son déroulement, deux catégories de données sont recueillies. Premièrement, des données d'observation de l'action : l'enregistrement vidéo de l'action des enseignants et des élèves à l'aide d'une caméra VHS sur pied et d'un micro HF par exemple. Deuxièmement, des données après l'action : l'enregistrement audio des verbalisations lors d'un entretien d'autoconfrontation a posteriori des enseignants au sujet de leur leçon par exemple. Ces entretiens d'autoconfrontation se déroulent immédiatement après l'action. L'auto-confrontation consiste à demander à l'acteur confronté à des traces de son action (ici l'enregistrement vidéo) de décrire, de montrer et commenter cette action
} 
bien que les grands discours n'ont aucun impact sur eux. Là je n'ai pas trop d'illusions, je les vois qui doivent se dire : “Ça recommence !”, mais s'il y en a trois ou quatre au total que ce discours peut secouer... Qui se disent : “Ho là ! Il a raison, il faut que je me bouge... ”. C'est pour cela que j'insiste un peu. C'est sûr que ces discours généralement glissent sur eux, et que c'est le travail au jour le jour qui les fait bouger... Des projets précis ils n'en ont pas vraiment, à part quelques-uns... Et là, je leur rappelle que plus leur bulletin est bon, moins il leur ferme de choix pour l'an prochain, au moins dans les matières principales... Je tiens à leur mettre en tête cette échéance. Là je crois, à voir la tête de certains, que ça les touche. Ça me surprend de les voir subitement attentifs... Je me dis : "Hé hé ! Ça a l'air de marcher cette alerte...". C'est bon à savoir."

L'engagement de cet enseignant est constitué des préoccupations ou intérêts immanents découlant de ses expériences passées. Cet engagement ouvre pour lui un champ de possibles susceptibles de se réaliser. Il correspond à des attentes structurées relatives à la situation à cet instant, lesquelles spécifient des anticipations plus globales en rapport avec ses préoccupations. L’engagement est aussi en rapport avec la convocation de connaissances issues des expériences passées. Ces trois catégories (préoccupations, attentes et connaissances) constituent une structure d'attente, c'est à dire une disposition à agir globale et dirigée, émergeant dans la situation. Cette disposition à agir représente ce qui, de la culture de cet enseignant, est utile pour lui, mobilisé par lui ici et maintenant en fonction de ce qui fait signe pour lui dans la situation ${ }^{3}$

Dans l'extrait ci-dessus, l'enseignant ancre son action sur certains aspects de la prise en main de la classe qui font signe pour lui, notamment : L'excitation des élèves et leur bronzage ; Le moment où l'on se situe dans l'année scolaire ; La proximité de l'échéance du conseil de classe. Sa capacité à les percevoir et à leur conférer une signification pour l'action a été construite au cours de ses expériences passées et exprime une partie de sa culture professionnelle mobilisée dans l'instant. De cet ancrage dans la situation présente émergent chez l'enseignant les préoccupations suivantes :

Intervenir dès l'entrée en classe des élèves; Se montrer gentil avec les élèves; Mettre la classe sans délai dans le tempo du travail scolaire ; Responsabiliser les élèves; Atténuer le caractère moralisateur du discours. Ses attentes sont : Que quelques élèves se montrent convaincus; Les élèves vont manifester un intérêt limité à l'égard de ce discours moralisateur. Les connaissances sont constituées d'unités telles que : Il faut parfois brusquer les élèves; Les discours grandiloquents sont peu efficaces; Le début du cours et la rentrée des vacances sont des moments particuliers; Les élèves ont tendance à relâcher leurs efforts ou à se démobiliser après une période de vacances; La deuxième partie du deuxième trimestre est une phase décisive pour l'orientation ; L'orientation en deuxième année de lycée se fait en fonction des résultats dans quelques matières principales; Il y a un relâchement au deuxième trimestre qui se traduit par une baisse des résultats; Les élèves travaillent au jour le jour et non en fonction d’un projet à long terme ; Le rôle des enseignants

pas à pas, lors d'un entretien guidé par le chercheur. Il lui est notamment demandé de restituer ce qu'il faisait, ce qu'il pensait, ce qu'il prenait en compte pour agir, ce qu'il percevait ou ressentait.

${ }^{3}$ A partir de Theureau (1992, 2000), la construction des données, permettant l'identification des catégories de l'expérience de l'acteur, est réalisée en réponse au questionnement suivant :

- Quels éléments perçus (ou rappelés) de la situation font signe pour l'enseignant à l'instant étudié ?

- Quelles sont les préoccupations de l'enseignant en fonction de ce qui fait signe pour lui dans la situation?

- Quelles sont les attentes de l'enseignant à l'instant étudié, prolongeant concrètement ses préoccupations ?

- Quelles sont les connaissances mobilisées par l'enseignant à l'instant étudié ?

- Quelle est l'action à un instant étudié, dont l'enseignant peut rendre compte a posteriori de façon significative?

- Quelles connaissances sont en cours de construction selon un processus de confirmation ou d'infirmation ? 
est d'aider les élèves; Les élèves doivent avoir à l'esprit les échéances de l'orientation; Une intervention qui touche trois ou quatre élèves dans la classe n'est pas négligeable.

Sans déterminer l'action de façon stricte et mécanique, ces composantes de la culture de cet enseignant constituent un cadre à partir duquel se développe l'action. Les unités d'action prennent un sens en rapport avec ces éléments culturels mobilisés lors de cette prise en main de la classe au retour des vacances : L'enseignant brusque un peu les élèves; Il rappelle l'échéance du conseil de classe ; Il insiste sur l'importance des notes dans les décisions d'orientation; Il ajuste son discours en classe en fonction des réactions des élèves; Il intervient gentiment en direction des élèves; Il propose son aide.

L'enseignant, en voyant la tête de certains élèves, perçoit leur réaction attentive à son discours : “ça les touche ". Cette courte séquence contribue (même modestement) à la construction de sa culture professionnelle. En effet, elle aboutit à une diminution de la validité de l'unité de connaissance : Les discours grandiloquents sont peu efficaces, et à une augmentation de la validité des unités : Les élèves ont tendance à relâcher leurs efforts ou à se démobiliser après une période de vacances; Il faut parfois brusquer les élèves.

Repli sur l'expérience personnelle et partage culturel

Singularité et communauté de l'enseignement

Bien qu'étant des acteurs publics, dont le travail est précisément de communiquer (Tardif et Lessard, 2000), les enseignants sont des solitaires : ils ont tendance à partager beaucoup moins leur expérience professionnelle que d'autres professions dont la nature du travail impose une activité collective (Engeström et Middleton, 1996). Une fois la porte de leur classe fermée, ils sont seuls face à leurs élèves. Et combien d'entre eux, durant des stages de formation à l'analyse des pratiques, se sont étonnés en s'adressant à leurs collègues du même établissement : “ Ah, toi, tu t’y prends comme cela! ”.

Néanmoins, il existe des facteurs favorisant la communauté et le partage dans cette profession. En premier lieu l'expérience vécue en tant qu'élève procure une familiarité des novices avec ce travail qui est sans aucune mesure avec une autre profession et dont on apprécie l'impact massif en cours de formation (Huberman, 1989 ; Nadot, 2000 ; Tardif et Lessard, 2000). Par ailleurs, des lieux et opportunités d'échange existent dans les établissements scolaires : conseils ou projets collectifs auxquels participent les professeurs (débats à l'occasion de questions d'actualités ou de réformes impulsées par le Ministère); et plus rarement mutualisation des compétences en formation initiale ou continue (observation d'autres enseignants au travail ou co-animation de classes, lecture de revues professionnelles).

En raison de cela et de l'uniformité des conditions de pratique, l'exercice fondamentalement solitaire de ce travail n'empêche pas l'existence d'éléments apparaissant comme des invariants structurant les pratiques et la culture professionnelle. L'observation détaillée d'enseignants différents révèle qu'ils réalisent en classe des actions similaires en dépit des différences d'une classe à l'autre, d'un professeur à l'autre, d'une discipline à l'autre (Durand, 1996, 2001). L'action est simultanément l'expression d'une individualité, d'une singularité et d'une communauté.

Ceci est également repérable au cours des entretiens de conseil en formation initiale des enseignants lors des changements de posture d'énonciation pendant les échanges verbaux. Chaque intervention du maître de stage et de l'enseignant en formation est liée à une dynamique de présentation de soi et de son engagement vis à vis du travail (Durand, 2000 ; Waite, 1995). L’implication personnelle transparaît dans les formules suivantes, extraites d'entretiens divers et qui concernent les enseignants en formation initiale : "Je savais que ça ne poserait pas de problème parce que ce sont des enfants habitués à travailler dans ce sens là ...”, ou leurs maîtres de stage : “ Je suis mon plan de cours mais sans être obsédé par ça hein... Je suis aussi... Aussi à l'affût des réactions des élèves... ”, “ Je préfère leur dire d'emblée ce que 
j'attends d'eux ". Cette implication de soi dans le discours va de pair avec une analyse pas à pas de la leçon, selon la chronologie de l'action au cours des entretiens de conseil.

Une autre position d'énonciation peut être adoptée par les enseignants en formation : “Nous ne savons pas comment nous y prendre là... Tu sais les stagiaires, nous avons un problème d'autorité... ”, “ C'est peut-être parce qu'on prépare trop les cours... Quand on débute... On cherche trop à suivre nos préparations... ”, et aussi par les maîtres de stage : “ On évite les affrontements de face comme ça... On a plutôt tendance, nous, à régler ça en douce... Enfin ça dépend des élèves... Mais nous ne cherchons pas l'épreuve avec ces élèves ", “ Il nous faut nous adapter à ces élèves nouveaux... Ils ne sont plus du tout attentifs, ils sont trop vivants et turbulents... Notre métier a changé ". Dans ces cas, le discours signale dans le même temps une exclusion et une inclusion : le " nous" (ou le " on ") positionne respectivement le novice ou le maître de stage comme des représentants d'une communauté (sans d'ailleurs que sa délimitation soit très précise) : les professeurs novices, les professeurs expérimentés, les professeurs de cette académie, de cet établissement... Ceci a pour conséquence d'exclure l'interlocuteur de cette communauté (j'en suis, tu n'en es pas). Dans ce cas, l'échange verbal au cours de l'entretien dépasse de beaucoup la simple communication entre personnes : ce sont deux communautés qui sont en contact.

Enfin, on observe aussi des énoncés au caractère impersonnel : “ Parce qu'il faut quand même que les élèves apprennent ", “Si l'on n’a pas réussi à mettre les élèves en projet, il est évident que ça n'a pas beaucoup de sens tout ça ”,

“Est-ce qu'il faut toujours les placer en situation de problème... Ne faut-il pas aussi leur dire comment faire de temps en temps ?". Ce degré de généralité fait que les deux protagonistes ne sont qu'indirectement impliqués, comme si les personnes, les actions, les communautés disparaissaient derrière des énoncés dépersonnalisés et décontextualisés. Ceci témoigne à la fois d'une abstraction et d'une sorte d'identification de l'acteur avec son métier, sa profession. C'est le signe d'un certain détachement de l'action in situ et d'un engagement professionnel dessiné par les contours de cette profession.

\section{Le repli sur l'expérience personnelle}

Quel que soit le secteur professionnel, il devient de plus en plus difficile de s'exprimer au nom d'un collectif en raison d'une évolution du travail. La maîtrise des processus de travail est devenue une question cruciale. Elle occupe une place particulière parmi les transformations qui affectent le monde de l'éducation. Par exemple, la figure traditionnelle de l'enseignant qui boucle le programme est remise en cause tant dans ses fondements que dans sa plausibilité. Le modèle taylorien, sous-jacent à la conception républicaine de l'école et du travail des enseignants, est en crise, et avec lui la séparation entre conception et exécution, la notion de prescription du travail, de découpage du travail en tâches discrètes, définies par des buts et des conditions stables de réalisation (Durand et Arzel, sous presse). L'enseignement comporte aujourd'hui une forte part d'incertitude, d'indétermination, tenant à la dynamique des savoirs, aux nouveaux modes de relation aux élèves et à la nécessité de justifier dans l'action un pacte éducatif sans cesse mis en cause en raison de l'indisponibilité a priori de sa signification (Durand, 1996). Aussi, le travail des enseignants, vécu comme énigmatique, est-il désormais défini en termes de missions et non plus de tâches (Durand, 1996 ; Tardif et Lessard, 2000). Dans le cas des missions, seuls les objectifs à atteindre sont (plus ou moins) déterminés, et ce sont les résultats obtenus qui sont appréciés. Or en matière d'enseignement, l'évaluation de l'atteinte des objectifs éducatifs est problématique en raison de la nature même de ces objectifs, de sorte que la régulation et l'évaluation de l'action sont nécessairement basées sur des critères endogènes peu objectifs. Les sociétés modernes, se caractérisant par une autonomie accrue des acteurs (Martucelli, 1999), engendrent davantage de responsabilité individuelle et, secondairement, des possibilités de culpabilité et de souffrance au travail (Dejours, 1993). De sorte que le rapport aux pratiques professionnelles, notamment des enseignants, est de plus en plus “ à distance ” de rôles sociaux déjà écrits et porteurs d'une signification sociale préétablie. 
Les acteurs, comme prix de leur autonomie, sont rabattus sur leur expérience personnelle et mis en situation d'inventer des procédures efficaces et de construire la signification de leurs pratiques. Le chercheur est lui aussi renvoyé à l'étude des expériences individuelles (Dubet, 1994), et à ce qui fait qu'au-delà de leur diversité, de leur singularité et de leur manque de cohésion a priori, on n'assiste pas à un émiettement des pratiques (Dubet, 2000).

\section{Genres et styles en enseignement}

L'action présente toujours des traits singuliers. Mais elle est aussi l'expression d'organisations qui la dépassent, et que, selon les auteurs, on dénomme cadres, types, structures archétypes, habitus, schèmes.... S'il est possible de comprendre ce que fait un enseignant, si son action n'a pas la plupart du temps un caractère d'étrangeté, c'est en raison de cette organisation sous-jacente (Quéré, 2000). La culture de tout enseignant apparaît marquée par une double articulation, à un niveau personnel et à un niveau collectif.

Ceci est particulièrement apparent lors des entretiens de conseil pédagogique au cours desquels les novices sont d'une part incités à construire des styles personnels et d'autres part confrontés aux composantes partagées et collectives du métier qu'on pourrait dénommer des types ou, à l'instar de Clot (1999), des genres professionnels. Ainsi par exemple les débuts de leçons (Clot et Soubiran, 1998) sont une phase de l'action des enseignants novices qui alimente une majorité d'entretiens de conseil pédagogique. Cette composante du travail enseignant répond à deux préoccupations principales : l'appel et le rappel (Durand, 2000). L’appel consiste pour les enseignants à s'assurer que tous les élèves sont présents, mais aussi que tous “y soient”, c'est à dire dans des dispositions propices au travail scolaire. Dans cette recherche de disponibilité des élèves, s'entremêlent des composantes opérationnelles et éthiques : être efficace et ne délivrer des consignes aux élèves que si l'on peut être entendu d'eux, établir une rupture avec les événements antérieurs (interclasse, déplacements dans la cour et les couloirs du collège, ambiance du cours précédent...), être équitable et ne pas commencer avant que tous les élèves ne soient présents, assurer un enseignement identique pour tous... Le rappel est aussi marqué par ce double souci opérationnel et éthique. Il s'agit de conférer à l'activité scolaire, qui suit cette entrée en matière, un sens accessible pour les élèves et de compenser les inconvénients associés à la structuration de leur travail en unités de temps administratives : les cours d'une à deux heures, les semaines, les trimestres, les années scolaires... Les enseignants s'efforcent d'atténuer ce caractère discontinu et segmenté de leur action, et de donner un sens au travail entrepris avec les élèves en convoquant des souvenirs du travail passé censé rétablir, chez eux, une continuité, en mettant la leçon du jour en rapport avec les grandes lignes du travail futur, et en l'instrumentalisant par rapport à l'atteinte d'objectifs pédagogiques ou de certification.

L'action des maîtres de stage en direction des enseignants novices prend la forme d'un accueil dans une communauté de pratiques (Lave, 1988), et parfois même d'une adoption (nous avons entendu un maître de stage dire à une jeune professeur de Français : “Nous sommes frère et sœur en pédagogie ”). Comme l'illustre le cas suivant, cet accueil n'a pas lieu dans une communauté professionnelle homogène. Cette jeune professeur de Français avait accepté de participer successivement, avec deux maîtres de stage différents, à deux entretiens de conseil ayant pour prétexte une leçon conduite par elle avec des élèves d'une classe de deuxième année de lycée. Ces deux entretiens font apparaître des désaccords importants.

Extrait de l'entretien de conseil avec le maître de stage ${ }^{\circ} 1$ (MS 1) : “ Je trouve que, à chaque séance tu devrais reprendre ce qu'on a dit à la séance précédente [...] Insister sur la démarche, le plan, le plan du cours, le plan de la séquence. [...] Il ne faut pas avoir peur d'insister, prendre le temps de bien justifier ta 
séquence, de manière à ce que les élèves comprennent ce qu'ils vont faire et se situent par rapport à la séquence précédente".

Extrait de l'entretien de conseil avec le maître de stage n² (MS 2) : “ Tu ne te débrouilles pas si mal : ça va vite, tu ne perds pas de temps en parlotes inutiles [...] Ce que tu fais là est assez proche de ce que je fais moi : on ne perd pas de temps, on s'assure que tout le monde y soit. Tant pis si ça les brusque un peu, qu'ils comprennent bien qu'on va démarrer un cours et que l'interclasse est fini [...] On les rappelle à l'ordre, on leur demande le silence, puis on s'assure que tous sont prêts avec leur matériel, on rappelle brièvement ce qui a été fait précédemment et hop on démarre avec tous les élèves qui y vont en même temps ".

Ces désaccords demeurent alors que les deux maîtres de stage ont, a posteriori, été invités à échanger à propos de la leçon de cette enseignante novice. Toutefois ce nouvel entretien fait aussi apparaître une recherche de consensus.

Extrait de l'entretien entre les deux maîtres de stage :

MS 2 : “... Je pense qu'il faut être très méticuleux en début de cours ...”

MS 1 : “... Je suis bien d'accord avec toi, ce n'est pas moi qui dirai le contraire..."

MS 2 : “ ... L'essentiel n'est pas tant dans la façon de faire que dans les finalités et là, je pense qu'on est bien d'accord tous les deux : il ne faut pas manquer ce moment un peu crucial, mettre très vite les élèves en activité, tout en leur rappelant où se situe le travail du jour par rapport aux leçons précédentes "

MS 1 : “ “...Tout en instaurant un climat de fermeté et de confiance (...). Prendre le temps de faire l'appel et le rappel de la séance précédente "

MS 2 : “Oui, oui nous sommes bien d'accords là dessus... D'accords pour dire que c'est important le début de la leçon. C'est un moment critique qu'il vaut mieux ne pas rater".

Ces accords permettent de repérer un genre professionnel (Clot, 1999) tandis que les désaccords portent sur le style personnel. Il y a consensus sur un mode de structuration et de découpage du travail et sur la signification de ce genre. Au-delà de ce sur quoi porte ce consensus, c'est son existence même qui est révélatrice d'une structuration culturelle de l'action professionnelle. En revanche, on se dispute sur les styles, sur les sensibilités individuelles. C'est à cette communauté et à ces singularités qu'est confrontée l'enseignante novice qui, à l'occasion de ces entretiens, prend connaissance des querelles de famille et aussi des éléments qui cimentent la culture enseignante. L'acquisition de compétences professionnelles se fait de façon complexe par la visite de genres professionnels reconnus (fût-ce sur un mode implicite) et la constitution d'un style propre dans un rapport mimétique ou d'opposition avec un mentor.

\section{Contagion des idées et formation}

\section{$\underline{\text { Registres de pertinence et transmission culturelle }}$}

Le partage d'une culture au sein d'une communauté implique au moins deux catégories de processus : la capitalisation de l'expérience personnelle et la transmission à d'autres membres de la communauté d'une partie de ce qui a été capitalisé. La transmission s'opère selon des mécanismes comparables à une épidémie ou une contagion dont on peut analyser les progrès de proche en proche (Nowack, Szamrej et Latané, 1990 ; Sperber, 1996 ; Vallacher et Novack, 1994). Les dispositifs de formation des enseignants constituent des situations privilégiées pour étudier ce processus de contagion. 
L'action des enseignants peut, à certains égards, être décrite comme un suivi de règles et les entretiens de conseil pédagogique comme des occasions d'expliciter ces règles. Par exemple l'énoncé Ne jamais sourire avant Noël, résume une règle de sévérité initiale et de relâchement de la discipline en cours d'année, qui schématise et guide l'action de nombreux enseignants (Shulman, 1987). La conformité ou non à cette règle constitue pour eux un critère d'évaluation de leur action. Se créent donc avec l'expérience chez ces enseignants, des blocs de connaissance tels qu'ils sont satisfaits de leur action, non pas s'ils réussissent à atteindre les objectifs éducatifs, mais s'ils agissent conformément à ces blocs de connaissance et si les événements ne sont pas incompatibles avec des attentes globales relatives à ce qu'est l'enseignement (Durand, 1996).

Le dispositif de conseil pédagogique, dans ce qu'il a d'efficace et aussi de contre-productif (Chaliès et Durand, 2000) permet d'apporter quelques indications quant à ces processus de transmission de ces connaissances. Dans son organisation typique ce dispositif alterne deux phases : au cours de la première l'enseignant novice conduit une leçon avec une classe en présence de son maître de stage qui l'observe ; au cours de la seconde, cette leçon fait l'objet d'un entretien de conseil. Durant l'entretien, la leçon est décrite, analysée, évaluée par les deux protagonistes à partir de leur propre culture, et en réalité essentiellement à partir de celle du maître de stage qui exerce une certaine forme de leadership au sein de la dyade.

Une partie importante de ces entretiens entre enseignants experts et novices lors des formations initiales consiste à confronter les novices à des règles d'actions et à tenter de les convaincre de les suivre. On observe fréquemment des conflits durant ces entretiens et une résistance des enseignants novices aux conseils qui leur sont prodigués. Cette résistance nous paraît pour partie liée à un double registre d'évaluation de l'action.

Pendant la classe, les novices construisent de nouvelles connaissances en action alors que la pression du résultat est élevée. Cette construction s’opère à partir de critères tangibles d'efficacité : essentiellement la discipline, le taux d'activité des élèves, le respect des plans de leçons (Durand, 1996). Est alors considérée comme valide une connaissance déterminant une action efficace, et invalide celle déterminant une action inefficace. Pendant l'entretien, lorsque les actions des novices sont évaluées par rapport à des critères pragmatiques et contextualisés, les interactions entre le maître de stage et le novice se déroulent de façon estimée efficace et lisse ; en revanche, lorsque les critères sont très généraux, décontextualisés, l'évaluation et les conseils sont rejetés par les novices qui déploient des stratégies d'évitement, de contournement, de dénégation... (Durand et Chaliès, 2001).

\section{Validation et invalidation des connaissances}

Le contexte cognitif au sein duquel se construisent les connaissances est, comme l'a analysé Peirce (1984), une sorte d'entre-deux allant du doute à la confiance. Dans la mesure où elle concerne une action professionnelle encore incertaine et ressentie comme complexe et menaçante par les enseignants novices, cette construction suppose l'acceptation d'un risque. Ce risque est inhérent à toute action humaine (Mendel, 1999), mais cette menace est plus élevée et fortement ressentie en contexte d'apprentissage dans l'action.

L'acquisition de connaissances professionnelles nouvelles repose sur des opérations élémentaires de renforcement de la validité de ce qui est efficace et d'invalidation de ce qui est inefficace (Durand, 2000 ; Sève, Saury, Theureau et Durand, sous presse). La construction s'opère par l'accroissement de la validité de chaque élément de connaissance ou encore l'émergence d'un élément nouveau ayant d'emblée une validité satisfaisante ; la destruction s'effectue par diminution de leur validité. Ces deux opérations s'entremêlent chez les acteurs au sein d'un complexe qui allie crédulité et conservatisme (Sève et al., sous presse). La crédulité est la tendance à valider de façon non suspicieuse une connaissance nouvelle dès la première échéance positive d'une action nouvelle. Elle est alors, par la suite, convoquée 
et détermine l'action future. Le conservatisme est la tendance à ce que, pour qu'une connaissance soit invalidée, il faut qu'elle soit mise en défaut de façon non ambiguë et répétée.

Ces caractéristiques générales de la cognition humaine marquent les processus de transmission culturelle des fonctions professionnelles. La résistance des enseignants novices aux injonctions de leurs maîtres de stage est peut-être liée à ce conservatisme. Ils hésitent à substituer le nouveau à l'ancien, tout en restant cependant prêts à attribuer du crédit à toute nouvelle connaissance.

La constitution d'un répertoire professionnel implique l'individu dans sa totalité et représente bien plus qu'un simple enregistrement de connaissances qui s'ajouteraient les unes aux autres. Chaque élément nouveau perturbe et nécessite des processus de rééquilibration. Par ailleurs chaque action étant singulière, elle ne peut être modifiée ou transformée de façon simple. A chaque fois est en jeu une dynamique d'assimilation à des formes partagées de pratiques (et donc de visite des genres professionnels) et d'affirmation d'un style personnel.

\section{Partages d'expériences et acculturation des enseignants novices}

Une des questions est de savoir comment l'expérience professionnelle de chaque enseignant peut devenir un objet de connaissance explicite et partageable pour d'autres.

Toute expérience est par essence difficilement explicite car souvent syncrétique, pré-réfléchie et inscrite dans un contexte particulier non renouvelable. La narration de l'expérience professionnelle constitue une des clefs du dévoilement de l'agir professionnel des enseignants, et particulièrement des novices. Mais il ne suffit pas de recourir à l'expression verbale de l'action et à la description du travail pour obtenir des garanties quant à la fiabilité de ce que les acteurs restituent. Au contraire, on sait depuis les travaux de sociologie des professions que la manière dont des professionnels décrivent leur travail, renvoie à des stratégies subtiles qui masquent la réalité du travail, et renforcent la tendance évoquée plus haut à un double registre d'énonciation : celui de l'action qui est largement implicite, bricolé, circonstanciel, et celui de la formalisation pour autrui qui est explicite, rationalisé et peu opérant.

C'est la raison pour laquelle, pour que les dispositifs de formation professionnelle basés sur la mise en récit ou la narration de son expérience professionnelle, aient une efficacité par rapport aux objectifs d'acculturation des enseignants novices, ils doivent satisfaire certaines conditions et être conçus de façon systématique. Ils doivent permettre à la narration de détacher la signification de l'événement de l'action (Ricœur, 1986) et de transformer l'expérience initiale. L'expérience dévoilée continue de vivre, d'évoluer et de s'enrichir à partir des échanges sociaux (avec des pairs ou un formateur) et devient une construction sociale et culturelle. Dans cette mesure, la narration est autant une action de transformation des novices que la restitution d'un récit de vie (Bertaux, 1997 ; Pineau et Legrand, 1993).

Trois dispositifs de formation (Ria, 2001), qui correspondent à la narration d'expériences singulières dans une visée d'acculturation, sont évoqués dans les lignes suivantes.

\section{Faire sienne l'expérience d'autrui}

L'enseignant en formation observe, décrit et analyse l'action d'un autre enseignant novice enregistré en vidéo en tentant d'exprimer ce qu'a dû être l'expérience de ce dernier à ce moment-là. Dans l'exemple qui suit, la vidéo concerne une enseignante novice qui a commis, en s'adressant à ses élèves, une erreur de grammaire en parlant de “ petits travails ”.

Formateur : "L'enseignante dit " travails", “des petits travails" et les élèves lui rétorquent “ des petits travaux" et elle sourit... D'après toi, comment l'enseignante vit la correction publique de cette petite faute ?... Là, tout de suite ?" 
Enseignant en formation : “ C'est dur de se faire reprendre par les élèves... On n'est forcément pas à l'aise par rapport à ça... C'est sûr que l'on doit essayer de parler correctement avec les élèves, faire attention à ce qu'on dit, et quand on se fait reprendre, et ben, ce n'est jamais bien agréable... Les élèves le prennent en souriant; elle aussi sourit, mais je ne pense pas que ce soit une situation très agréable pour elle, on est mal à l'aise dans ce cas... Il faut essayer de parler correctement. J'essaie de parler correctement devant les élèves, de ne pas faire de fautes, parce que, pour eux, quand même, on reste l'enseignant... Et l'enseignant n'a souvent pas le droit à l'erreur avec eux... Là pour elle cela reste gentil, mais après ce n'est pas facile d'enchaîner sans être perturbé ".

Les connaissances mobilisées pour interpréter la situation d'enseignement sont ici : C'est inconfortable de se faire reprendre par les élèves lors de la présentation d'une consigne ; Quand on est perturbé il est difficile “d'enchaîner ” face aux élèves... Cette interprétation de l'expérience d'autrui contribue à la fois à mobiliser les traces de sa propre expérience et à percevoir le caractère potentiellement partageable d'expériences considérées jusque là intimes, circonstancielles et peu intéressantes pour autrui. L'usage des pronoms “ Je ”, “ Elle ”, “ Il ” ou encore “ On ” indique à la fois des éléments d'analyse liés à son expérience, et plus largement aux expériences-types des novices lors de la présentation de consignes en classe.

\section{Faire autre sa propre expérience à partir de l'expérience d'autrui}

L'enseignant en formation est invité à décrire ce qu'il aurait fait à la place de l'enseignante novice d'Education Physique dont l'action a été visionnée initialement. Cette enseignante conduisait un exercice d'étirement des muscles des jambes au début d'une leçon de Volley Ball et s'était engagée dans une procédure consistant à demander aux élèves d'effectuer cet exercice tout en écoutant des consignes relatives à l'exercice à venir.

Formateur : "Donc toi, tu ferais comment ici, là ? Si tu étais à ce moment dans la situation ? Est-ce que tu donnes des consignes ici pendant que les élèves s'étirent ?"

Enseignant en formation : "Heu... Pendant que mes élèves s'étirent ?"

Formateur : "Oui..."

Enseignant en formation : “Pendant qu'ils s'étirent... Non ! Je reste sur les étirements... C’est à dire que je ne leur explique pas la suite.... Et une fois que j'ai fini mes étirements, là... Je leur présente la suite..." Formateur : "Et... Si tu essayais de faire les deux en même temps?"

Enseignant en formation : “ Hum !... [Silence]. Si je devais pendant mes étirements présenter la suite...

[Silence]. Je pense que je continuerais à démontrer [les étirements], c'est à dire que je leur donnerais des consignes du type : “Faut se placer comme ça ” et après je leur dirais : “ A vous ! " Et pendant qu'ils seraient en train de le faire, là, je pourrais leur dire : "Vous voyez... Vous allez vous organiser comme ça, là... Pour ce qui va suivre "... Donc priorité à ma consigne sur l'étirement et peut-être un petit temps entre deux consignes d'étirement pour leur expliquer l'organisation de ce qui va suivre ”.

L'enseignant en formation est critique vis à vis de l'action de l'enseignante observée. Son interprétation critique découle de sa propre expérience d'enseignement avec des élèves. Pourtant, contre son gré au début, il propose des solutions se traduisant par l'alternance d'actions relatives au contrôle des élèves et à la présentation de consignes dans des intervalles de temps très courts. La confrontation à l'action d'autrui est une opportunité pour élargir le répertoire des 
interventions possibles pour ce type de situations : l'enseignant en formation propose à la fois des solutions potentiellement efficaces pour l'enseignante observée et pour lui-même.

\section{L'instruction à un sosie : Faire autre sa propre expérience}

L'enseignant en formation est invité à instruire un " sosie " virtuel en lui donnant les consignes utiles pour qu'il soit en mesure de le remplacer dans son travail sans que personne ne s'avise de la substitution. Le sosie résiste à la description et au partage de la version de l'activité donnée par l'instructeur, en se montrant parfois récalcitrant pour que ce dernier précise, parmi les alternatives possibles, la genèse de ses choix. Il doit aussi exiger une double description : celle de la situation d'enseignement et celle de la conduite à tenir dans cette situation (Clot, 1999). Dans l'extrait suivant, l'instruction porte sur la façon de présenter les consignes à la classe en Education physique.

Sosie (Formateur) : “ Je ne connais pas du tout les élèves de ta classe, et je n'ai pas d'expérience en tant qu'enseignant, j'aimerais que tu me donnes des conseils pour que je puisse être efficace avec ta classe... Pour que je puisse m'en tirer. Donc, les élèves se sont déjà échauffés et je dois leur présenter la première consigne collective par rapport à ta première situation ..."

L'enseignant en formation : "Il faut aller vite ! Mais il vaut mieux perdre un peu de temps pour que cela soit très précis... N'hésite pas non plus à marquer au tableau la consigne... Ça va perdre effectivement un peu de temps, mais tu auras un peu plus l'assurance qu'ils aient compris... Donc perdre du temps au départ peut éviter, même si ce n'est pas toujours le cas, de revenir après dessus et de perdre du temps pour voir qu'ils n'ont pas compris... Il faut alors les regrouper à nouveau, reposer des questions pour voir qu'ils n'ont pas compris. C'est une sécurité, quoi !”

Sosie (Formateur) : “ Mais... Si j'ai des élèves qui ne comprennent pas la situation, qu'est-ce que je fais ?" L'enseignant en formation : "Si vraiment tu sens qu'il y a un flottement, que c'est trop compliqué, à ce moment-là, tu démontres l'exercice"

Sosie (Formateur) : “C'est à dire ?"

L'enseignant en formation : “Ça dépend des fois. Tu montres comme cela doit se dérouler sur le terrain, tu expliques en même temps, tu te sers du tableau, et après, si vraiment tu vois qu'ils n'ont pas compris, tu démontres seul ou avec des élèves..."

Sosie (Formateur) : "Comment voit-on que les élèves ne comprennent pas ?"

L'enseignant en formation : “A leur tête : les regards dans le vague, les yeux baissés, les élèves qui se font des signes, des débuts de discussion... On sent qu'il y a un flottement..."

Sosie (Formateur) : “Si j'ai l'impression que ça s'est plus ou moins bien passé, qu'un doute subsiste par rapport à la compréhension de l'exercice... Qu'est-ce que je fais ? "

L'enseignant en formation : “Euh, moi, j'ai tendance à passer... C'est à dire qu'une fois que j'ai lancé les élèves, je passe dans les groupes pour vérifier : je pose la question à un élève pour voir s'il a compris, puis un autre... Si tu t'aperçois qu'il y a vraiment un gros problème, tu arrêtes tout et tu reprends... Là c'est frustrant, il faut arrêter tout le monde et recommencer...".

Ce dispositif permet l'exploration, par l'enseignant novice, de sa propre expérience. Apparaissent ses repères en classe : Les regards; Les yeux baissés... Les connaissances qu'il mobilise dans l'action : La compréhension, par les élèves de l'exercice à réaliser prime sur le gain de temps; Il est nécessaire de recourir à la démonstration pour lever toute 
ambiguïté... Est souligné en même temps le caractère contradictoire, conflictuel de son action : “ Gagner du temps pendant la consigne " et " Perdre du temps pour expliquer l'action des élèves ".

Le dévoilement de l'expérience pour la rendre explicite et partageable entraîne son détachement vis-à-vis de la situation d'enseignement dans laquelle elle s'est développée. Elle s'en trouve dédoublée, c'est à dire à la fois dévoilée mais encore dépassée et transformée par le biais de la confrontation avec autrui.

Ces trois extraits montrent succinctement comment des dispositifs de formation, par l'explicitation de sa propre expérience et la confrontation à celle des autres, peuvent contribuer à la transformation de l'agir professionnel des enseignants novices. Ce lieu d'échange social contribue ainsi à l'élargissement des styles de chacun tout en constituant la mémoire interpersonnelle de leur communauté de pratiques.

\section{Conclusion}

Analyser simultanément la cognition et la culture en action des enseignants est un programme de recherche en perspective. Ce programme n'est viable que si les chercheurs dans le domaine de la cognition acceptent de développer des recherches en situation (et qui prennent au sérieux cette situation) et si les sociologues acceptent de se préoccuper de situations locales (et qui prennent au sérieux cette localité). Ceci ne signifie pas qu'il faille occulter les influences et les déterminants macro-sociologiques ou psychologiques sur ces actions situées : nul ne saurait raisonnablement nier que l'école est une institution obéissant à des dynamiques sociales qui la dépassent, ni que les élèves sont des personnes disposant d'attributs (aptitudes, traits de personnalité, bagage cognitif...) rendant plus ou moins faciles leurs trajectoires scolaires. Mais le présupposé selon lequel les situations et les actions en situation disposent d'une autonomie essentielle, même si elle est limitée, nous paraît de nature à relancer les recherches relatives aux connaissances des enseignants et à leur culture professionnelle. Ce programme de recherche pourrait se structurer en trois composantes qui correspondent aux trois thèmes développés dans cet article : les processus d'actualisation et de construction des connaissances dans l'action, les relations entre le caractère singulier des actions en classe et la possibilité d'une culture partagée, et les processus de transmission au sein de la communauté enseignante. Il reste sans aucun doute à faire preuve de créativité pour dépasser le dilemme énoncé par Jean Lave, mais un tel programme a à nos yeux, entre autres mérites, celui de positionner la question de la formation des enseignants à la fois dans une perspective cognitive et culturelle.

\section{Références}

Becker, H.S., Carper, J.W. (1956). The elements of identification with an occupation. American Sociological Review, $\underline{21}(3), 341-347$.

Becker, H.S., Geer, B., Riesman, D., Weiss, R. (1968). Institution and the person : Essays in honor of Everett Hughes. Chicago, Il. : The Falmer Press.

Bertaux, D. (1997). Les récits de vie. Paris : Nathan.

Bredo, E. (1994). Reconstructing educational psychology : Situated cognition and Deweyian pragmatism. Educational Psychology, 29(1), 23-35.

Brown, J.S., Collins, A., Duguid, P. (1989). Situated cognition and the culture of learning. Educational Researcher, 18(1), $32-42$.

Carraher, T.N., Carraher, D.W., Schliemann, A.D. (1985). Mathematics in the streets and in the schools. British Journal of Developmental Psychology, 3, 21-29.

Chaiklin, S., Lave, J. (1993). Understanding Practice : Perspectives on Activity and Context. Cambridge : Cambridge University Press. 
Chaliès, S., Durand, M. (2000). L'utilité discutée du tutorat en formation initiale des enseignants. Recherche et Formation, $35,145-180$.

Clot, Y. (1999). La fonction psychologique du travail. Paris : PUF.

Clot, Y., Soubiran, (1999). “Prendre” la classe : une question de style ? Société Française, 62/63, 78-88.

Dejours, C. (1993). Travail usure mentale. Paris : Editions Bayard.

Dreyfus, H. (1986). Intelligence artificielle : mythes et limites. Paris : Seuil

Dubar, C., Tripier, P . (1998). Sociologie des professions. Paris : Armand Colin.

Dubet, F. (1994). Sociologie de l'expérience. Paris : Seuil.

Dubet, F. (2000). Rôle et expérience. In J.M. Barbier (Ed.), L’analyse de la singularité de l'action (pp. 71-82). Paris : PUF Durand, M. (1996). L’enseignement en milieu scolaire. Paris : PUF.

Durand, M. (2000). Développement personnel et accès à une culture professionnelle en formation initiale des professeurs. In C. Gohier et C. Alin, (Eds), Enseignant-Formateur. La construction de l'identité professionnelle (pp. 67-83). Paris : L'Harmattan.

Durand, M. (2001). Chronomètre et survêtement. Reflets de l'expérience quotidienne d'enseignants d'Education Physique. Paris : Editions Revue EPS.

Durand, M., Arzel, G. (sous presse). Autonomie et connaissance dans les conceptions de l'apprentissage, de l'enseignement et de la formation des maîtres. In M. Altet, M. Carbonneau, P. Perrenoud, M. Tardif (Eds.), Réforme scolaire et formation des enseignants. Bruxelles : De Boeck.

Durand, M. et Chaliès, S. (2001). Le conseil pédagogique : partage d'expérience et transmission culturelle. In Journée d'Etude d'Anthropologie. Clermont Ferrand, CNRS.

Engeström, Y., Middleton, D (Eds.) (1996). Cognition and communication at work. Cambridge : Cambridge University Press.

Gal-Petitfaux, N., Durand, M. (2001). L'enseignement de l'Education physique comme “ action située ” : propositions pour une approche d'anthropologie cognitive. STAPS, 55, 79-100.

Greeno, J.G., The Middle School Mathematics Through Application Project Group (1998). The situativity of knowing, learning and research. American Psychologist, 53, 1, 5-26.

Huberman, M. (1989). La vie des enseignants : évolution et bilan d'une profession. Neuchatel : Delachaux et Niestlé.

Hughes, E.C. (1958). Men and their work. Glencoe : the Free Press.

Hutchins, E.A.(1995). Cognition in the wild. Cambridge : The MIT Press.

Kirshner, D., Whitson, J. A. (Eds.) (1997) Situated Cognition : Social, semiotic, and psychological perspectives. Mahwah, NJ : Erlbaum.

Lave, J. (1988). Cognition in practice : Mind, mathematics and culture in everyday life. Cambridge : Cambridge University Press.

Lave, J., Wenger, E. (1991). Situated learning : Legitimate peripheral participation. Cambridge : Cambridge University Press.

Leblanc, S., Saury, S. Sève, C., Durand, M., Theureau, J. (2001). An analysis of a user's exploration and learning of a multimedia instruction system. Computers \& Education, 36, 59-82.

Leplat, J. (2000). L'environnement de l'action en situation de travail. In J.M. Barbier (Ed.), L'analyse de la singularité de l'action (pp. 107-131). Paris : PUF.

Martucelli, D. (1999). Sociologies de la modernité. Paris : Gallimard

Mendel, G. (1999). L'acte est une aventure. Paris : La Découverte. 
Nadot, S. (2000). Acquérir une pratique professionnelle d'enseignant. Un nécessaire changement dans le rapport au savoir.

In C. Gohier et C. Alin, (Eds), Enseignant-Formateur. La construction de l'identité professionnelle (pp. 111-124).

Paris : L'Harmattan.

Nowack, A., Szamrej, J., Latané, B. (1990). From private attitude to public opinion : A dynamical theory of social impact.

Psychological Review, 97, 362-376.

Peirce, C.S. (1978). Ecrits anticartésiens. Paris : Aubier

Peirce, C.S. (1984). Ecrits sur le signe. Paris : Editions du Seuil.

Pineau, G. et Legrand, J.L. (1993). Les histoires de vie. Paris : PUF.

Quéré, L . (2000). Singularité et intelligibilité de l'action. In J.M. Barbier (Ed.), L'analyse de la singularité de l'action (pp. 147-170). Paris : PUF

Ria, L. (2001). Les préoccupations des enseignants débutants en Education Physique et Sportive. Etude de l'expérience professionnelle et conception d'aides à la formation. Thèse de doctorat STAPS non publiée. Université de Montpellier 1.

Ria, L., Saury, J., Sève, C., Durand, M. (2001). Les dilemmes des enseignants débutants : Etudes lors des premières expériences de classe en Education Physique. Science et Motricité, 42, 47-58.

Ricoeur, P. (1986). Du texte à l'action. Paris : Seuil.

Rosch, E. (1973). On the internal structure of perceptual and semantic categories. In T.E. Moore (Ed.), $\underline{\text { Cognitive }}$ development and the acquisition of language (pp. 111-144). New York : Academic Press.

Schliemann, A. D., Carraher, D.W. (1992). Proportional reasoning in and out of school. In P. Light \& G. Butterworth (Eds), Context and cognition : Ways of learning and knowing (pp. 47-73). New York : Harvester Whestsheaf.

Sève, C., Saury, J., Theureau, J. Durand, M. (sous presse). L'analyse de l'activité à visé de performance de pongistes professionnels, Le Travail Humain.

Shulman, L.S. (1987). Knowledge and teaching : Foundation for a new reform. Harvard Educational Review, 51, 1-22.

Sperber, D. (1996). La contagion des idées. Paris : Odile Jacob.

Still, A., Costall, A. (Eds.)(1991). Against cognitivism. Alternative foundations for cognitive psychology. New York : : Harvester Wheatsheaf.

Suchman, L. (1987). Plans and situated actions : The problem of human-machine communication. Cambridge : Cambridge University Press.

Tardif, M., Lessard, C. (2000). Le travail enseignant au quotidien. Bruxelles : Editions De Boeck.

Theureau, J. (1992). Le cours d'action. Berne : Peter Lang.

Theureau, J. (2000). Anthropologie cognitive et analyse des compétences. In J.M. Barbier (Ed.), L'analyse de la singularité de l'action (pp. 171-211). Paris : PUF.

Thévenot, L. (1990). L'action qui convient. In P. Ladrière, P. Pharo, L. Quéré (Eds), La théorie de l'action. Le sujet pratique en débat (pp.39-69). Paris CNRS.

Vallacher, L., Nowack, A. (Eds.) (1994). Dynamical systems in social psychology. San Diego, CA : Academic Press.

Varela, F. (1989). Autonomie et connaissance. Paris : Seuil.

Varela, F.J., Thompson, E., Rosch, E. (1993). L’inscription corporelle de l'esprit. Paris : Seuil.

Waite, D. (1995). Rethinking instructional supervision : Notes on its language and culture. New prospects series : 1. London : The Falmer Press. 
La culture en action des enseignants

Whitson J.A. (1997). Cognition as a semiotic process : From situated mediation to critical reflective transcendence. In D.

Kirshner et J.A. Whitson (Eds), Situated cognition : Social, semiotic and psychological perspectives, (pp.

97-149). Mahwah, NJ : Erlbaum Associates.

Winograd, T., Florès, F. (1989). L'intelligence artificielle en question. Paris : PUF. 